\title{
Frequency-dependent shot noise in single-electron devices
}

\author{
V. Talbo, J. Mateos, S. Retailleau*, P. Dollfus*, and T. González \\ Dpto. Física Aplicada, Universidad de Salamanca, Plaza de la Merced s/n, 37008 Salamanca, Spain \\ *Institut d'Electronique Fondamentale, Univ. Paris-Sud, CNRS UMR 8622, F-91405 Orsay, France \\ e-mail: vincent.talbo@gmail.com
}

\begin{abstract}
The simulation of a double-tunnel junction with the SENS simulator gives access to the frequency-dependent and static behavior of shot noise. The concept of basic paths in a multi-state process provides a clear interpretation of the noise regimes, and allows locating cut-offs in autocorrelation functions and spectral densities.
\end{abstract}

Keywords-Single-electron device; Double-tunnel junction; Shot Noise; Monte-Carlo simulations.

\section{INTRODUCTION}

Shot noise $(\mathrm{SN})$, consequence of the granularity of electron charge, has been intensively investigated within the last decades [1]. Coulomb blockade devices, where electrons are flowing through tunnel barriers one by one, are thus of great interest for SN studies.

To characterize noise, its spectral density $S(f)$ is usually compared to the spectral density of a Poissonian process $2 q\langle I\rangle$. The ratio at zero frequency $F=S(0) / 2 q\langle I\rangle$ is called Fano Factor. If $F<1(>1), \mathrm{SN}$ is sub-(super-) Poissonian. If $F=1, \mathrm{SN}$ is Poissonian.

The home-made simulator SENS (Single-Electron Nanodevices Simulation) has been used previously to analyze the behavior of $\mathrm{Si}$ quantum dot (QD)-based double-tunnel junctions (DTJs) [2] and single-electron transistors (SETs) [3]. It consists in the self-consistent solution of 3D PoissonSchrödinger equations, depending on the bias and number of electrons in the dot. Tunnel transfer rates are computed from the resulting wave functions and used in a Monte-Carlo (MC) algorithm or the master equation coupled with Korotkov's method [4] to determine the electronic characteristics, such as current and SN.

Korotkov's method is very powerful to quickly reach accurate autocorrelation functions and frequency-dependent spectral densities. However, the physics at the origin of the SN behavior is hidden within a complex mathematical formalism.

In contrast, the $\mathrm{MC}$ algorithm, though time consuming, allows us to follow the time evolution of the system. According to the number of electrons in the QD and the corresponding transfer rates, the time between two events is randomly picked.

This work has been partially supported by the Dirección General de Investigación Científica y Técnica (MINECO) through Project TEC201341640-R and by the Junta de Castilla y León through Project SA052U13.)
Then, the tunnel event is also randomly chosen (an electron goes in or out of the QD). A glance at resulting time/current characteristics, as well as a comparison between tunnel transfer rates, helps to understand the behavior of shot noise.

In this work, the frequency-dependent and static SN are presented for a given DTJ. Their behavior is explained by considering transfer rates and characteristic times for each transition in a simple 3-state case.

\section{RESULTS AND DISCUSSION}

\section{A. Overall results}

The studied structure is a DTJ consisting in a $10 \mathrm{~nm}$ QD, with $1.2 \mathrm{~nm}(1.8 \mathrm{~nm})$ source (drain) oxide thicknesses. The study has been made at $0 \mathrm{~K}$ to avoid the contribution of thermal noise. At this temperature, we can only consider source-to-dot (dot-to-drain) tunnel transfer rates, written $\Gamma_{\text {in }}(N)\left(\Gamma_{\text {out }}(N)\right)$, where $N$ is the number of electrons in the dot before the tunnel transfer.

In Fig. 1, the time evolution of the number of electrons going through source barrier shows a situation known as "bunching", i.e., a lot of events happen in a short time, followed by a long time without any event. This is the signature of a super-Poissonian SN.

The current autocorrelation functions for three different regimes (sub-Poissonian, super-Poissonian and close to Poissonian transport) are shown in Fig. 2. MC calculations fit nearly perfectly with Korotkov's method. The autocorrelation function remains positive (negative) for super-(sub-) Poissonian Fano factor. Close to the Poissonian case, the autocorrelation function goes from negative to positive values before reaching zero.

The spectral densities of current $S(f)$ are then calculated from the autocorrelation functions. The corresponding results are given in Fig. 3. For all cases, spectral densities are given by the Fano factor at low frequency and the Poissonian regime $(S(f)=2 q\langle I\rangle)$ is finally reached at high frequency. Remarkably, in the case of a Fano factor close to 1, the transport becomes sub-Poissonian in the $\left[10^{5} \mathrm{~Hz}-10^{8} \mathrm{~Hz}\right]$ range, thus indicating the presence of some specific dynamics in such frequency range associated to the values of the transfer rates. 


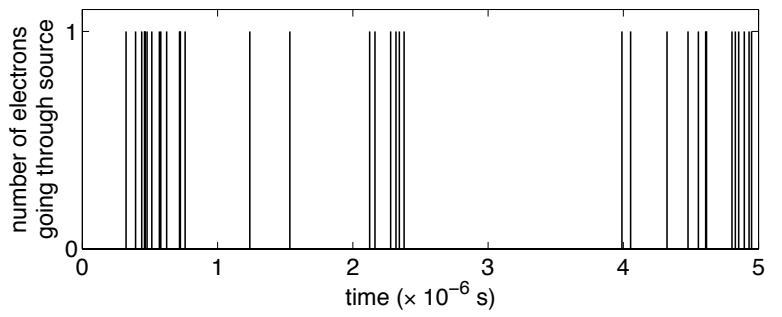

Fig. 1. Time evolution of the number of electrons going through the device in a case of a super-Poissonian noise $F=4.67$. The mean current is $0.76 \mathrm{pA}$.

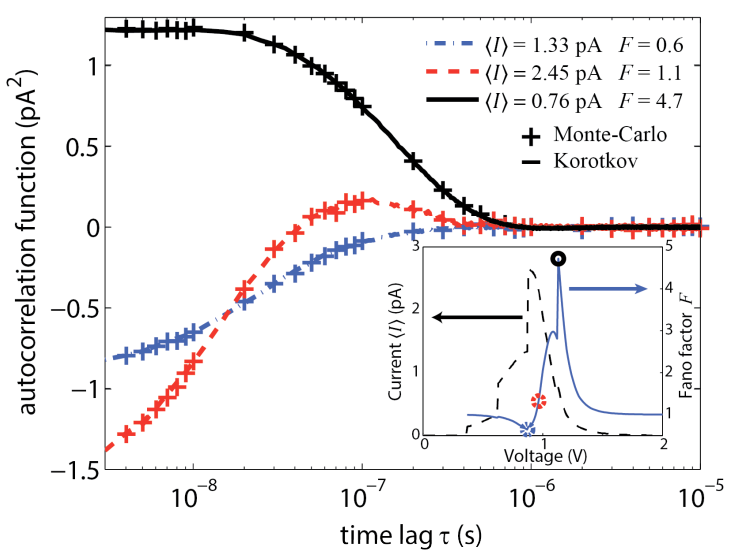

Fig. 2. Current autocorrelation function for super-, sub- and Poissonian noise. Inset: Current-voltage and Fano factor-voltage characteristics for a given DTJ. The Fano factors studied are indicated by circles. The value at $\tau=0$ (variance) is not shown for clarity.

The close to Poissonian and super-Poissonian regimes are reached respectively on the $3^{\text {rd }}$ and the $4^{\text {th }}$ steps of the Coulomb staircase, as shown on the inset of Fig. 2 . Thus, respectively 6 and 8 tunnel transfer rates are to compare $\left(\Gamma_{\text {in }}(N=0,1,2)\right.$ and $\Gamma_{\text {out }}(N=1,2,3)$ on the $3^{\text {rd }}$ step), making the analysis of noise behavior difficult. Hence, we will consider a simple 3-state case (i.e. with a maximum of 2 electrons in the QD), which can generate super-Poissonian transport.

\section{B. Analysis of noise in a 3-state case}

In a previous article [5], the zero frequency superPoissonian noise has been explained through the study of the tunnel transfer rates in a 3-state case (0-1-2 electrons in the QD) schematized in Fig. 4a. Any evolution of the number of electrons in the dot is a sequence built with 4 basic paths: (0)101, (1)212, (1)2101 and (0)12, sketched in Fig. 4b. These basic paths represent the sequence we can observe between two tunnel events through the source barrier. After an electron has entered through the source barrier, each path has a probability $P_{\text {path }}$ and a characteristic time between two current peaks $t_{\text {path }}$, depending only on the tunnel transfer rates. Their study allows us to analyze the frequency behavior of SN.

In particular, we focus our attention on the two extreme values of the Fano factor of the 3-state case. The values of path probabilities and characteristic times are given in Table I.

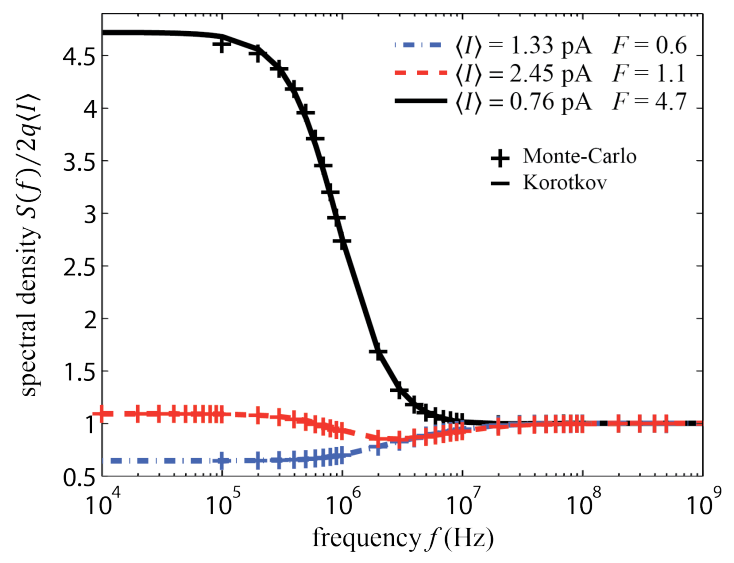

Fig. 3. Spectral density of current as a function of frequency obtained in the case of sub-Poissonian, super-Poissonian and Poissonian Fano factors.

(a)

(b)
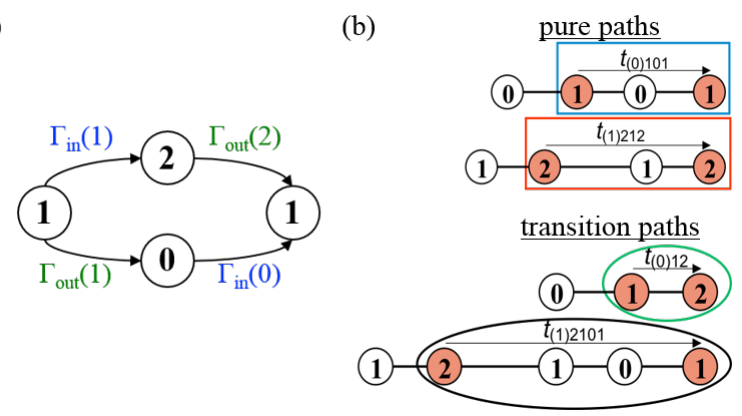

Fig. 4. Schematic description of (a) all possible transitions in a 3-state case, and (b) basic paths followed by the number of electrons in the QD between two tunnel events through source oxide (red circles). The numbers in circles are the number of electrons in the quantum dot.

TABLE I. PROBABILITIES AND CHARACTERISTIC TIMES OF BASIC PATHS FOR THE EXTREME VALUES OF THE FANO FACTOR

\begin{tabular}{|c|c|c|c|c|}
\hline \multirow{2}{*}{$\begin{array}{c}\text { Basic } \\
\text { paths }\end{array}$} & \multicolumn{2}{|c|}{$\boldsymbol{V}=\mathbf{0 . 9} \boldsymbol{F}=\mathbf{0 . 6 2}$} & \multicolumn{2}{c|}{$\boldsymbol{V}=\mathbf{1 . 1 5} \mathbf{F}=\mathbf{1 . 4 2}$} \\
\cline { 2 - 5 } & $\boldsymbol{P}_{\text {path }}$ & $\boldsymbol{t}_{\text {path }}$ & $\boldsymbol{P}_{\text {path }}$ & $\boldsymbol{t}_{\text {path }}$ \\
\hline 0101 & $2 \%$ & $10^{-7} \mathrm{~s}$ & $36 \%$ & $6 \times 10^{-7} \mathrm{~s}$ \\
\hline 1212 & $70 \%$ & $10^{-7} \mathrm{~s}$ & $16 \%$ & $10^{-7} \mathrm{~s}$ \\
\hline 012 & $14 \%$ & $3 \times 10^{-8} \mathrm{~s}$ & $24 \%$ & $6 \times 10^{-8} \mathrm{~s}$ \\
\hline 12101 & $14 \%$ & $2 \times 10^{-7} \mathrm{~s}$ & $24 \%$ & $6 \times 10^{-7} \mathrm{~s}$ \\
\hline
\end{tabular}

$\boldsymbol{F}=\mathbf{0 . 6 2}$ : In the case of sub-Poissonian transport, the main path is the 1212 , at $70 \%$. If the only path followed were the 1212 , the Fano factor would be $F=0.6$, using the calculation of a 2-state process [6].

However, the "transition paths" 012 and 12101 also occur with a rate of $14 \%$. Their characteristic times are twice slower and three times faster than the 1212 path, respectively. Thus, when an 12101 path occurs instead of an 1212, one event is "lost". Nevertheless, a fast 012 path must follow to reach again the 1212 process: the lost event due to the 12101 is almost compensated by the fast 012 . SN increases slightly, reaching $F=0.62$. 


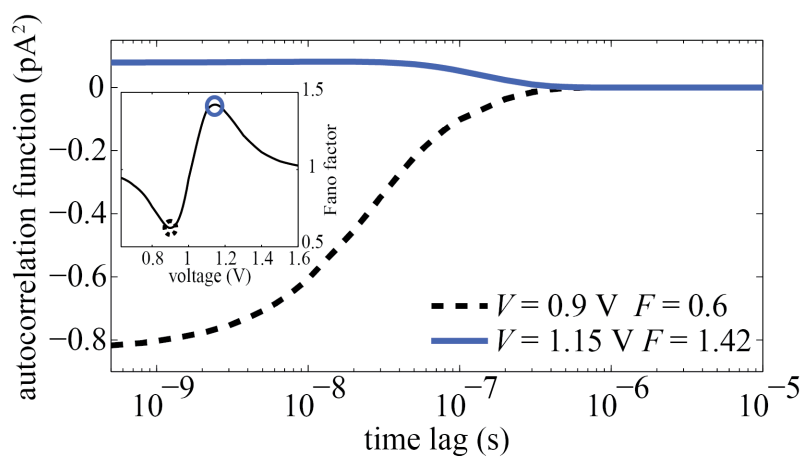

Fig. 5. Current autocorrelation function for the extreme values of $\mathrm{SN}$ in a 3-state case. Inset: Fano factor-voltage characteristic in a 3-state case. The Fano factors studied are indicated by circles.

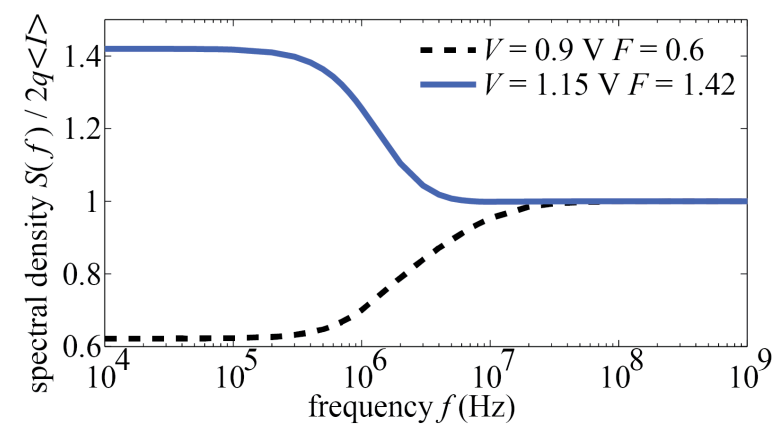

Fig. 6. Spectral density of current as a function of frequency obtained for the extreme values of the 3 -state case.

$\boldsymbol{F}=\mathbf{1 . 4 2}$. Because of the proximity of $\Gamma_{\text {in }}(1)$ and $\Gamma_{\text {out }}(1)$, basic paths have close probabilities (0101 at $36 \%$, transition paths at $24 \%$ ), thus every path is likely to happen. However, the 0101 and 12101 paths are 6 times and 10 times slower than 1212 and 012, respectively. This induces a bunching phenomenon, with fast events (1212 or 012) followed by slow events (0101 or 12101). This effect enhances the noise, to reach $F=1.42$.
Frequency behavior and characteristic times. As seen on the general results, the autocorrelation functions at low time lags, given in Fig. 5, show an anticorrelation in a case of a subPoissonian noise; a correlation for super-Poissonian. Cut-offs occur around a time lag corresponding the slowest basic path characteristic time, i.e, $t_{12101}$. After the cut-off, there is no correlation between events.

The same analysis can be done for spectral density, shown in Fig. 6. Here, the cut-offs occur close to a frequency equal to $1 / t_{012}$, where $t_{012}$ is the characteristic time of the fastest path.

\section{CONCLUSION}

Thanks to the simulator SENS, and the possibility to have access to the tunnel transfer rates for each configuration of number of electron in the QD, we have been able to simulate and interpret the frequency dependent and static SN in a DTJ. The concept of basic paths introduced in a 3-state case provides a clear explanation of static SN behavior, and allows us to localize the cut-offs in the autocorrelation functions and spectral densities, linked to the slowest and the fastest basic paths, respectively.

\section{REFERENCES}

[1] Ya. M. Blanter, M. Büttiker, "Shot noise in mesoscopic conductors," Phys. Rep., vol. 336, pp. 1-166, 2000.

[2] J. Sée, P. Dollfus and S. Galdin-Retailleau, "Theoretical investigation of negative differential conductance regime of silicon nanocrystal singleelectron devices," IEEE Trans. Electron Devices, vol. 53, pp. 1268-1273, 2006.

[3] V. Talbo, A. Valentin, S. Galdin-Retailleau and P. Dollfus, "Physical simulation of Silicon nanocrystal-based single-electron transistors," IEEE Trans. Electron Dev., vol. 58, pp. 3286-3293, 2011.

[4] A. N. Korotkov, "Intrinsic noise of the single-electron transistor," Phys. Rev. B, vol. 49, pp. 10381-10392, 1994.

[5] V. Talbo, D. Querlioz, S. Retailleau and P. Dollfus, "Sub- and superPoissonian in $\mathrm{Si}$ quantum dots using fully self-consistent 3D simulation," Fluct. Noise Lett., vol. 11, 1242006, 2012.

[6] S. Hershfield, J. H. Davies, P. Hyldgaard, C. J. Stanton and J. W. Wilkins, "Zero-frequency current noise for the double-tunnel-junction Coulomb blockade," Phys. Rev. B, vol. 47, pp. 1967-1979, 1993. 